\title{
Cochrane Review Summary: Interventions for improving outcomes in patients with multimorbidity in primary care and community settings
}

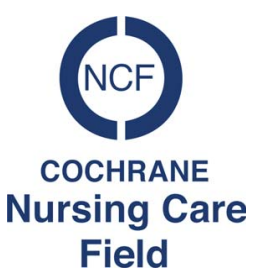

\section{Daksha Trivedi}

Senior Research Fellow, Centre for Research in Primary and Community Care, University of Hertfordshire, Hatfield, UK

Key words: comorbidity; community; intervention; multimorbidity; primary care

First published online 20 December 2016

\section{Review question}

Do health service or patient-oriented interventions improve outcomes for people with multimorbidity in primary care and community settings?

\section{Relevance to primary care and nursing}

The National Institute for Health and Clinical Excellence (NICE) guidelines highlight the need to provide tailored approaches and specialist services for people with multimorbidity. Primary health-care professionals such as GPs, pharmacists and nurses are involved in the clinical assessment, management and care of people with multiple morbidity (NICE, 2016).

\section{Characteristics of the evidence}

Included studies targeted people with multimorbidity, defined as two or more chronic conditions in the same person. The term comorbidity referred to combinations of defined conditions that commonly exist, for example, diabetes and heart disease. This Cochrane review contained 18 randomised-controlled trials (RCTs) of which nine targeted people with a broad range of conditions or multimorbidity (Smith et al., 2016). The remaining nine included comorbidities: depression with

Correspondence to: Dr Daksha Trivedi, Senior Research Fellow, Centre for Research in Primary and Community Care, University of Hertfordshire, College Lane, Hatfield, AL10 9AB, UK. Email: d.trivedi@herts.ac.uk

(C) Cambridge University Press 2016 hypertension, headache, diabetes and/or heart disease and one study with a sub-group of people who had at least two of diabetes, chronic obstructive pulmonary disease and irritable bowel syndrome.

Most studies evaluated interventions involving changes to the organisation of care delivery with some studies delivering more patient-focussed interventions. Interventions were delivered in primary care or community settings and compared with usual care. In all, 16 studies were conducted in the United States, one in the United Kingdom and one in Canada. Studies that did not aim to specifically target multimorbidity were excluded.

\section{Summary of key evidence}

Included studies had 8727 participants in total ranging from 50 to 4023 . Duration of intervention for most studies was 6-12 months, ranging from eight weeks to two years. Follow-up periods varied from immediate to 12 months post-intervention. RCTs were overall judged to be of reasonable quality with minimal risk of bias and a moderate certainty or confidence in the results, using GRADE (Grading of Recommendations Assessment, Development and Evaluation) criteria. Narrative synthesis and meta-analysis were conducted where appropriate, although results were heterogeneous. Outcomes included objective-validated measures and standardised effect sizes (SES) are given where available for effective outcomes (SES intervention effects: $>0.2=$ small $; \quad>0.5=$ moderate $; \quad>0.8=$ large $)$. Mean difference (MD) and 95\% confidence intervals (CIs) are given where appropriate. 
Organisational interventions included case management and coordination of care or the enhancement of skill mix in multidisciplinary teams in addition to patient care. Patient-oriented interventions included educational or group-based self-management support courses.

\section{Clinical outcomes}

These included glycaemic control and risk factors (HbA1c, blood pressure, cholesterol) in comorbidity studies. There was no evidence overall suggesting an effect on these outcomes (11 studies, SES 0.01-1.6). Management of risk factors in comorbidity was more likely to be effective with marginal improvement in systolic blood pressure [five studies, $n=892$; MD -3.10 (95\% CI -7.26 to 1.06)], reduction in cholesterol (SES 0.26, relative difference $9 \%, n=214$ ) and an improvement in combined risk factors (blood pressure, HbA1c, cholesterol) (relative difference 68\%) in studies that examined these outcomes.

\section{Mental health outcomes}

Evidence from nine studies suggested improvements in depression outcomes in comorbidity studies [SES 0.09-2.24, four studies $>0.5$; six studies, $n=1062$; MD -0.41 (95\% CI -0.63 to -0.20$)$ ], with no significant effect on anxiety.

\section{Patient-reported outcome measures}

The results from 12 studies reporting these outcomes were mixed with less than half reporting some benefit (SES 0.03-1.7, four studies $>0.5$ health-related quality of life) with no significant effect on other psychosocial outcomes.

\section{Health service use}

Evidence from five studies showed mixed effects.

\section{Patient behaviour}

Evidence is mixed for medication use and adherence (four studies) and other health-related behaviours such as diet, smoking and exercise (seven studies).

\section{Provider behaviour}

Five studies reported overall improvement, particularly in relation to care delivery, such as appropriate referrals $(n=3$ studies, $15-40 \%$ improvement).

\section{Implications for practice}

Although the evidence suggests that organisational interventions can improve depression outcomes in comorbidity studies, there is a need to develop interventions that target problems that occur across conditions such as functional disability for people with multimorbidity. There is also a need to consider relevant outcomes for multimorbidity studies and risk factor management in comorbidity to improve health outcomes and to support policy and practice.

\section{Implications for research}

Research needs to address how multimorbidity and comorbidity are defined, so that high-quality evaluations targeting appropriate interventions and outcomes can be developed. They need to consider the various components of organisational interventions, the theoretical assumptions and link to outcomes that can be used for different combination of diseases.

\section{Acknowledgements}

The author is a member of the Cochrane Nursing Care Field (CNCF).

\section{Financial Support}

This research received no specific grant from any funding agency, commercial or not-for-profit sectors.

\section{Conflicts of Interest}

None. 


\section{References}

National Institute for Health and Clinical Excellence (NICE) 2016: Multimorbidity: clinical assessment and management, NICE guideline. Retrieved 24 October 2016 from nice.org.uk/guidance/ng56.
Smith, S.M., Wallace, E., O'Dowd, T. and Fortin, M. 2016: Interventions for improving outcomes in patients with multimorbidity in primary care and community settings. Cochrane Database of Systematic Reviews, Issue 3, Art. No. CD006560. https://doi.org/10.1002/14651858.CD006560. pub3. 